\title{
Effectiveness of serological markers of eosinophil activity in monitoring eosinophilic esophagitis
}

\author{
Joaquín Rodríguez-Sánchez ${ }^{1,2}$, Elisa Gómez-Torrijos ${ }^{1,3}$, Eva de-la-Santa-Belda ${ }^{1,4}$, Bartolomé López- \\ Viedma $^{1,4}$, Francisco Martín-Dávila ${ }^{1,5}$, John Patrick Pilkington-Woll ${ }^{1}$, Paloma Donado-Palencia ${ }^{1,6}$, Pilar \\ Sánchez-Miranda ${ }^{1,6}$ and José Olmedo-Camacho ${ }^{3}$
}

${ }^{I}$ Eosinophilic Esophagitis Work Group. HGUCR/HGOV. Ciudad Real, Spain. ${ }^{2}$ Department of Gastroenterology. Gutiérrez Ortega Hospital. Valdepeñas, Ciudad Real. Spain. Departments of ${ }^{3}$ Allergology, ${ }^{4}$ Gastroenterology, ${ }^{5}$ Pathology, and ${ }^{6}$ Pediatrics. Hospital General Universitario de Ciudad Real. Ciudad Real, Spain

\begin{abstract}
Background: it is necessary to find serological markers accessible in clinical practice to prevent the need to perform repeated endoscopies.

Objective: to assess the efficacy of eosinophil activity markers in monitoring eosinophilic esophagitis (EoE).

Material and methods: thirty patients were included prospectively, all under dietary treatment -diets excluding 6 foods, and allergy test based diet (skin prick test and specific IgE). The variables assessed were demographic parameters, eosinophil cationic protein $(\mathrm{ECP})$ levels $(\mu \mathrm{g} / \mathrm{mL})$, total IgE $(\mathrm{KU} / \mathrm{L})$, peripheral blood eosinophils (PBE) $\left(\mathrm{U} / \mathrm{mm}^{3}\right)$, and the maximum peak of eosinophils/hpf in esophageal biopsies. The variation found between these figures was assessed in line with response to dietary treatment.

Results: thirty patients (66.7 \% males; mean age 33.43 years) were included in the study, 22 responders and 8 non-responders. Ninety percent presented a personal history of atopy. No significant decrease was detected in serum total IgE and ECP after diet in responder and nonresponders. However, the PBE decreased significantly in responders but not in nonresponders, PBE in responders (pre-diet. 397.27 vs. post-diet 276.81, $p=0.024$ ) and non-responders PBE (pre-diet. 460 vs. post-diet 317.5, $\mathrm{p}=0.23$ ).

Conclusion: serum total IgE and ECP do not act as markers for EoE activity. However PBE may play a role in this regard, bearing in mind that this parameter may be influenced by concomitant atopic conditions.
\end{abstract}

Key words: Eosinophil cationic protein. Eosinophilic esophagitis. Biomarkers. Total serum IgE.

\section{ABBREVIATIONS}

ECP: Eosinophil cationic protein.

Received: 26-03-2013

Acepted: 11-09-2013

Correspondence: Joaquín Rodríguez Sánchez. Department of Gastroenterology. Hospital Gutiérrez Ortega de Valdepeñas. Avda. de los Estudiantes, s/n. 13300 Valdepeñas, Ciudad Real. Spain

e-mail: joakinrodriguez@gmail.com
ELSA: Eosinophilic esophagitis live symptoms assessment. Eo/hpf: Eosinophils/high power field.

GERD: Gastroesophageal reflux disease.

PBE: Peripheral blood eosinophils.

Total IgE: Total immunoglobulin E.

\section{INTRODUCTION}

Eosinophilic esophagitis (EoE) is an emerging condition characterized by symptoms of esophageal malfunction (primarily dysphagia and food impaction in adults), caused by a chronic inflammatory infiltration in the wall of the esophagus with high levels of eosinophils ( $\geq 15$ eosinophils/high power field), excluding other causes of esophageal eosinophilia (1). It is largely associated with atopic conditions such as asthma, allergic rhinoconjunctivitis, and atopic dermatitis (2). EoE is currently the second most common cause of chronic esophagitis behind gastresophageal reflux disease, and the main cause of food impaction in young patients (3).

It has recently been published how the six-food elimination diet (SFED) is effective in the induction and maintenance of remission after 3 years in the Mediterranean population (4). This provides an attractive treatment strategy for this condition, although it involves the performance of numerous endoscopies in order to monitor histological activity.

In order to identify serological markers for this disease, serum total IgE levels have been studied with varying results, increased levels being found in active disease in a highly variable proportion of cases (5). However, eosino-

Rodríguez-Sánchez J, Gómez-Torrijos E, de-la-Santa-Belda E, López-Viedma B, Martín-Dávila F, Pilkington-Woll JP, DonadoPalencia P, Sánchez-Miranda P, Olmedo-Camacho J. Effectiveness of serological markers of eosinophil activity in monitoring eosinophilic esophagitis. Rev Esp Enferm Dig 2013;105:462-467. 
phil derived proteins such as eosinophil derived neurotoxin (EDN) and major basic protein (MBP), and the mean peripheral blood eosinophils (PBE) count have proven to be significantly higher in patients with EoE, both in serum and histological samples (EDN and MBP). Eosinophil cationic protein (ECP) in serum has proven to be effective in the clinical monitoring of asthma and allergic rhinoconjunctivitis (6). However, its role in EoE has not been studied in depth.

The aim of our study was to analyze the use of total $\operatorname{IgE}$ $(\mathrm{KU} / \mathrm{ml}), \mathrm{PBE}\left(\mathrm{U} / \mathrm{mm}^{3}\right)$ and serum ECP levels $(\mu \mathrm{g} / \mathrm{mL})$ in the monitoring of histological activity of this condition.

\section{MATERIAL AND METHODS}

\section{Study design}

Adolescent and adult patients diagnosed with EoE were included consecutively as per criteria under current consensus (1) $[\geq 15$ eosinophils/high power field (eo/hpf) in esophagus biopsies, taking two from each arm after treatment with proton pump inhibitors (PPIs) for 8 weeks]. At the first visit all patients included in the study were sent, after the first diagnostic endoscopy, for ECP, serum total $\mathrm{IgE}$ and blood count (PBE) analysis, together with a basic food allergy study: Specific IgE (ImmunoCAP250. Phadia, Uppsala. Sweden) and Skin Prick Test (Alk-Abelló, Madrid. Spain) for food allergens (milk, egg, nuts, hake, prawn, lentil, wheat). Based on the results of the above tests, two diet-based treatment strategies were employed depending on sensitivity to foods: 19 sensitized patients were treated with a diet drawn up based on allergy testing, targeted diet (TD) and 11 non-sensitized patients underwent a 6-food elimination diet (SFED) (free of milk, egg, cereals, legumes, nuts, fish and shellfish). After 6 weeks on this diet, patients underwent a further esophagoscopy, taking 2 esophageal biopsies by sections (distal, medial and proximal). On the same day as the endoscopy, blood count (PBE), serum total IgE and ECP tests were repeated. Based on response to diets (SFED + TD), the sample was divided into responders $(\mathrm{R})$ (maximum peak $<15 \mathrm{eo} / \mathrm{hpf}$ ) and non-responders (NR) (maximum peak $\geq 15 \mathrm{eo} / \mathrm{hpf}$ ) comparing blood count results for total IgE, ECP and PBE, pre and post diet.

\section{Clinical activity of the condition}

The clinical condition of patients was assessed using the ELSA-VASEoE index (eosinophilic esophagitis live symptoms assessment). This is an unofficial tool, specific to EoE, which measures the subjective perception of the most common symptoms of the condition using a visual analogue scale, with scores ranging from 0 to 70 points (7). Assessment via this index was completed twice: The first upon confirming the diagnosis of the condition and the second after dietary treatment for 6 weeks.

\section{Endoscopic study}

Endoscopic procedures were performed by an experienced gastroenterologist using a flexible $9 \mathrm{~mm}$ gastroscope with a $2.8 \mathrm{~mm}$ work channel (GIF-Q160. Olympus. Hamburg, Germany). Biopsies were taken using conventional forceps (Radial Jaw ${ }^{\mathrm{TM}} 4$ Boston Scientific. Proparck, Costa Rica). No complications were observed as a result of this endoscopic procedure or the collection of biopsies.

Findings were classified into phenotypes, according to the endoscopic signals observed. In line with the progression of the condition in the extent of remodeling the esophagus wall the classification, from minor to major was: Inflammatory phenotype -those patients with longitudinal fissures, edema, whitish exudate and frail mucous membranes, stenosing phenotype where pseudo-rings and stenosis is observed, and mixed phenotype where the patient presented signs of inflammation and stenosis (8). In order to analyze the relationship between endoscopic findings and activity parameters, we divided the sample into three groups according to the improvement observed in the endoscopy after the diet: Improvement, no change, or worsening.

\section{Histological study}

Biopsy specimens were sent to the Pathology Department, where they are formalin-fixed and paraffin-embedded; serial sections were then made between 3 and $5 \mu \mathrm{m}$ using a microtome, and stained with eosin hematoxylin. Samples were examined under a Nikon ${ }^{\circledR}$ Eclipse 80i microscope (Nikon, Tokyo. Japan). Samples were also PAS diastase stained in order to rule out the presence of fungal infection. A diagnosis of "histologically compatible with EoE" was made if over 15 eosinophils per high power field, and in particular when the eosinophilic infiltrate was prevalent in the upper layers of the epithelium and clustered in microabscesses (the hpf area was $0.24 \mathrm{~mm}^{2}, \mathrm{x} 400$ magnification).

\section{Statistical analysis}

For the statistical analysis, the statistics software package PASW18 was used (SPSS. Chicago, IL. USA). The details of continuous variables (eo/hpf, ECP, PBE, total $\mathrm{IgE})$, were expressed in mean $\pm \mathrm{SD}$ and the Wilcoxon rank test was used for contrasting hypotheses in continuous variables for pre and post diet. The Chi-squared test was used for categorical variables. In order to assess the relationship between continuous variables the Pearson linear correlation coefficient was used. For all these analyses the 
statistical signification was established for an alpha error of $\leq 0.05$.

\section{Ethical aspects}

The study was designed in accordance with the principles of the Helsinki Declaration. All patients included in the study were required to sign an informed consent document, and the signatures of legal guardians were required for patients aged under 16. The study was assessed and approved by the IEC (Independent Ethics Committee) at the General University Hospital in Ciudad Real.

\section{RESULTS}

\section{Epidemiological data}

Thirty patients diagnosed with EoE (20 males and 10 females) from March 2012 to February 2013, aged between 14 and 73 (33.43; SD 13.65 years) were included consecutively. The epidemiological variables are summarized on table I.

\section{Clinical data}

The most common atopic pathology was asthma, presented by half the sample. It is notable that 12 patients presented subclinical atopy, i.e. sensitivity to both pneumoallergens and food, but did not present symptoms of atopy (asthma, rhinoconjunctivitis, atopic dermatitis). The most commonly used treatment strategy was a TD (specific $\operatorname{IgE}+$ Prick test), performed in $63 \%$ of patients, with an overall response rate to treatment with diets (TD + SFED) of $73.3 \%$ [TD response rate of $84.2 \%(16 / 19)$ and SFED $54.5 \%(6 / 11)]$. Considering these results, 22 responding patients $(\mathrm{R})$ and 8 non-responding patients were compared, both groups being homogenous in terms of the parameters studied, including the clinical situation prior to treatment, measured using the ELSA index (Table II).

\section{Endoscopic findings}

Upon analyzing the changes taking place in endoscopic findings bearing in mind the persistent signs of re-shaping and their connection to serological activity parameters, we detected that PBE is significantly lower in patients presenting an improvement in endoscopic findings (365.50 vs. 266.87; $\mathrm{p}=0.015$ ), although we also observed a drop in $\mathrm{PBE}$ in patients not presenting changes in comparison with the pre-diet endoscopy (551.25 vs. 332.50; $\mathrm{p}=0.017)$. No significant variation in other activity parameters was detected (Table III).
Table I. Characteristics of patients included in the study

\begin{tabular}{|c|c|}
\hline & Total \\
\hline Number of patients & 30 \\
\hline \multicolumn{2}{|l|}{ Epidemiological data } \\
\hline Men/Women & $\begin{array}{c}20 / 10 \\
(66.7 \% / 33.3 \%)\end{array}$ \\
\hline Age & $33.43 \pm 13.65$ \\
\hline Atopic patients & $28(93,3 \%)$ \\
\hline Sensitivity to pneumoallergens & $23(76.6 \%)$ \\
\hline Food allergies & $19(63.3 \%)$ \\
\hline Family history of atopy & $17(56.7 \%)$ \\
\hline \multicolumn{2}{|l|}{ Clinical data } \\
\hline Asthma & $15(50 \%)$ \\
\hline Rhinoconjunctivitis & $13(43.3 \%)$ \\
\hline Atopic dermatitis & $4(13.3 \%)$ \\
\hline Subclinical atopy (non-clinical allergy) & $12(40 \%)$ \\
\hline Eosinophil peak in biopsies (n/hpf) & $39.20 \pm 24.93$ \\
\hline $\begin{array}{l}\text { Naïve patients (no prior corticoid } \\
\text { treatment) }\end{array}$ & $18(60 \%)$ \\
\hline Pre-diet ELSA & $28.10 \pm 10.97$ \\
\hline Diet treatment (TD/SFED) & $63 \% / 36.7 \%$ \\
\hline Overall response to diet (TD + SFED) & $22(73.3 \%)$ \\
\hline \multicolumn{2}{|l|}{ Endoscopic findings } \\
\hline $\begin{array}{l}\text { Inflammatory phenotype (edema, fissures, } \\
\text { whitish spotting) }\end{array}$ & $10(33.3 \%)$ \\
\hline $\begin{array}{l}\text { Stenosing phenotype (pseudo-rings, } \\
\text { stenosis) }\end{array}$ & $5(16.7 \%)$ \\
\hline Mixed phenotype & $14(46.7 \%)$ \\
\hline Normal endoscopy & $1(3.3 \%)$ \\
\hline \multicolumn{2}{|l|}{ Analytical values } \\
\hline Total IgE levels (KU/L) & $385.65 \pm 354.67$ \\
\hline $\mathrm{ECP}(\mu \mathrm{g} / \mathrm{mL})$ & $38.59 \pm 27.13$ \\
\hline $\operatorname{PBE}\left(\mathrm{U} / \mathrm{mm}^{3}\right)$ & $414 \pm 214.88$ \\
\hline
\end{tabular}

ECP: Eosinophil cationic protein; PBE: Peripheral blood eosinophils; TD: Targeted diet; SFED: Six-food elimination diet.

\section{Analytical values}

At baseline, all patients presented high levels of total IgE, ECP and PBE (Table I). When parameters were analyzed according to response to diet, it was found that neither serum total IgE nor ECP presented a significant variation between $\mathrm{R}$ and NR. However, the PBE presented a significant drop in $\mathrm{R}$ (pre-diet $397.27 \pm 192.53 \mathrm{U} / \mathrm{mm}^{3}$ vs. post-diet $\left.276.81 \pm 145.48 \mathrm{U} / \mathrm{mm}^{3} ; \mathrm{p}=0.02\right)$, which was not detected in NR (pre-diet $460 \pm 277.12 \mathrm{U} / \mathrm{mm}^{3} v s$. post-diet $317.5 \pm 169.51 \mathrm{U} / \mathrm{mm}^{3} ; \mathrm{p}=0.23$ ). However, upon analysis of the relationship between the number of eo/hpf and post-diet PBE, no significant correlation $(r=0.15 ; p$ 
$=0.41)$ was found, as is the case for the remaining parameters. Serum total $\operatorname{IgE}(r=-0.07 ; \mathrm{p}=0.70)$ and $\mathrm{ECP}(\mathrm{r}=$ $-0.07 ; p=0.70$ ). Upon comparison between the activity parameters themselves, we observed a strong positive correlation between PBE and ECP (pre-diet $r=0.64 ; \mathrm{p}=0.00$ and post-diet $r=0.74 ; p=0.00)$, which was not observed in the case of serum total $\mathrm{IgE}$, which bore no correlation to either ESP (pre-diet $\mathrm{r}=0.17 ; \mathrm{p}=0.34$ and post-diet $r=0.12 ; p=0.52$ ), or ECP (pre-diet $r=0.08 ; p=0.65$ and post-diet $r=0.04 ; p=0.80)$. When the relationship between the ELSA score and serological activity markets was studied, no significant correlation was observed (PBE: $\mathrm{r}=0.27 ; \mathrm{p}=0.13$, serum total IgE: $\mathrm{r}=0.02 ; \mathrm{p}=0.90$, ECP: $0.07 ; \mathrm{p}=0.69$ ).

\section{DISCUSSION}

The study of activity parameters of EoE measured after dietary treatment constitutes a novel approach, as until now this assessment has taken place after treatment with corticoids (9). Using food elimination diets as treatment is a means of acting on the cause of the illness, thus removing the primary stimulus behind it and therefore all variation found in the parameters of activity will be the result of EoE remission or not.

For many years, numerous studies have been attempting to find markers for EoE activity which help to monitor the condition without the need for esophagus biopsies. With regard to total IgE, high levels have been observed in

Table II. Comparison of responders (R) and non-responders (NR)

\begin{tabular}{|c|c|c|c|}
\hline & Responders & Non-responders & $p$ value \\
\hline No. of patients: & 22 & 8 & \\
\hline \multicolumn{4}{|l|}{ Epidemiological data } \\
\hline Male/Female (\%) & $59.1 / 40.9$ & $87.5 / 12.5$ & 0.14 \\
\hline Age (y) & $33.82 \pm 14.34$ & $32.38 \pm 12.40$ & 0.79 \\
\hline Atopy & $20(90.9 \%)$ & $8(100 \%)$ & 0.37 \\
\hline Sensitivity to pneumoallergens & $17(77.3 \%)$ & $6(75 \%)$ & 0.89 \\
\hline Food sensitivity & $16(72.7 \%)$ & $3(37.5 \%)$ & 0.07 \\
\hline Asthma & $10(45.5 \%)$ & $5(62.5 \%)$ & 0.40 \\
\hline Rhinoconjunctivitis & $9(40.9 \%)$ & $4(50 \%)$ & 0.65 \\
\hline Atopic dermatitis & $4(18.2 \%)$ & $0(0 \%)$ & 0.19 \\
\hline Subclinical atopy & $10(45.5 \%)$ & $2(25 \%)$ & 0.31 \\
\hline Family history of atopy & $13(59.1 \%)$ & $4(50 \%)$ & 0.65 \\
\hline \multicolumn{4}{|l|}{ Clinical findings } \\
\hline Eosinophil peak in biopsies (prediet) (n/hpf) & $39.59 \pm 23.58$ & $38.13 \pm 30.08$ & 0.89 \\
\hline Eosinophil peak in biopsies (postdiet) (n/hpf) & $1.91 \pm 2.48$ & $33.75 \pm 12.47$ & $<0.001$ \\
\hline Pre-diet ELSA & $29.55 \pm 9.69$ & $24.38 \pm 13.91$ & 0.26 \\
\hline \multicolumn{4}{|l|}{ Pre-diet endoscopic findings } \\
\hline Inflammatory phenotype (edema, fissures, whitish spotting) & $7(31.8 \%)$ & $3(37.5 \%)$ & 0.91 \\
\hline Stenosing phenotype (pseudo-rings, stenosis) & $4(18.2 \%)$ & $1(12.5 \%)$ & 0.89 \\
\hline Mixed phenotype & $10(45.5 \%)$ & $4(50 \%)$ & 0.80 \\
\hline Normal endoscopy & $1(4.5 \%)$ & $0(0 \%)$ & 0.79 \\
\hline \multicolumn{4}{|l|}{ Post-diet endoscopic findings } \\
\hline Inflammatory phenotype (edema, fissures, whitish spotting) & $2(9.1 \%)$ & $1(12.5 \%)$ & 0.78 \\
\hline Stenosing phenotype (pseudo-rings, stenosis) & $6(27.3 \%)$ & $3(37.5 \%)$ & 0.58 \\
\hline Mixed phenotype & $0(0 \%)$ & $3(37.5 \%)$ & 0.002 \\
\hline Normal endoscopy & $14(63.6 \%)$ & $1(12.5 \%)$ & 0.013 \\
\hline \multicolumn{4}{|l|}{ Analytical values } \\
\hline Total IgE levels (KU/L) & $399.43 \pm 387.42$ & $347.82 \pm 261.94$ & 0.73 \\
\hline $\mathrm{ECP}(\mu \mathrm{g} / \mathrm{mL})$ & $34.82 \pm 19.60$ & $48.96 \pm 41.59$ & 0.21 \\
\hline $\operatorname{PBE}\left(\mathrm{U} / \mathrm{mm}^{3}\right)$ & $397.27 \pm 132.53$ & $460 \pm 277.12$ & 0.48 \\
\hline
\end{tabular}

ECP: Eosinophil cationic protein; PBE: Peripheral blood eosinophils. It is observed that the groups are homogenous in all the parameters analyzed. 
Table III. Relation between serological markers of activity and post-diet endoscopic findings based on esophageal wall remodeling parameters

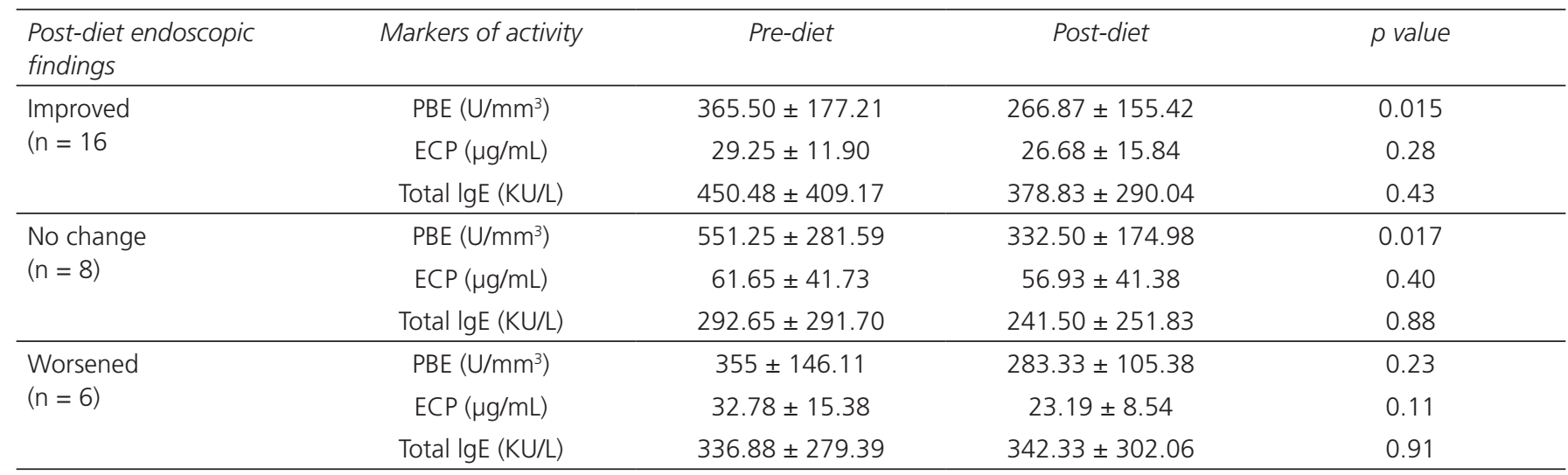

ECP: Eosinophil cationic protein; PBE: Peripheral blood eosinophils.

between $4 \%$ and $69 \%$ of adults with EoE and $33 \%$ to $71 \%$ of pediatric patients (5). However, its role as a marker for EoE activity is limited, just as in our study, Baxi et al. (10) did not find any correlation between total IgE levels and the number of eosinophils found in esophagus biopsies. Nevertheless, it has been demonstrated that $\operatorname{IgE}$ is produced in the esophageal epithelium of patients with EoE (11), but this local production is insufficient to cause a significant increase at peripheral level. There is ever increasing evidence that EoE does not behave in the same way as a purely IgE-mediated allergic reaction, but rather more like a Th2 lymphocyte-mediated reaction (12). This can be seen in the fact that the raised levels of serum total $\operatorname{IgE}$ in serum have not proven to act as predictive factors for response to treatment with corticoids (9). In studies published with diets used in the adult population, a weak correlation has been found between the foods responsible for reactivation of the condition and the results of allergy testing, which translates into an IgE-mediated reaction to food allergens (Prick test and specific $\operatorname{IgE}$ ) $(4,13)$. As regards its role as a marker for activity in other conditions such as asthma, a recent study detected how IgE behaves as a poor predictor for the presence of eosinophils in sputum (14), which in turn have proven to be a reliable parameter for the monitoring of asthma (15). It is therefore interesting to note the lack of correlation found between total $\mathrm{IgE}$ and the other two parameters studied (PBE and $\mathrm{ECP}$ ), which may indicate the huge influence of bronchial hyperreactivity and asthma on total $\mathrm{IgE}$ levels in serum on an ongoing basis (16), bearing in mind that in our study, $50 \%$ of patients are asthmatic, and $73.3 \%$ present sensitivity to pneumoallergens. Consequently, and given that between 40 and $70 \%$ of patients with EoE present concomitant asthma (1), it seems logical to conclude that total IgE does not act as a useful serological marker for EoE activity.

Evidence of the role of eosinophil degranulation products as serological markers is limited; eosinophil-derived neurotoxin (EDN) being the most studied parameter to have prov- en its use as a marker in both feces and serum (17). MBP has been studied at histological level by Dellon et al. (18) demonstrating that there is an increased expression of this protein in biopsies taken from EoE patients in comparison with patients with GERD. However, despite being a widely established marker in the management of atopic conditions, ECP has practically not been assessed in the management of EoE. Just as in our study, Chehade et al. (19) found increased levels of serum ECP in EoE patients as compared to a control group, despite not presenting serum eosinophilia. This study describes a significant correlation between peripheral eosinophilia and serum ECP levels, which has also been observed in our patients. However, this correlation was not obtained from the eosinophil count in esophagus biopsies, as also occurred in the study by Straumman et al. (9) where ECP figures did not bear any relation to the drop in number of eo/hpf detected after treatment with budesonide. This is fundamental, as it demonstrates that increased level of ECP is not directly connected to EoE activity, but is probably caused by concomitant atopic conditions, which in our study was the case in $93.3 \%$ of patients. However, at local level, ECP may be of use in this regard, as can be seen in the work of Furuta et al. (20) where, through the use of a device known as the Esophageal String Test, high levels of intraluminal ECP are observed in patients with active EoE in comparison with those with inactive EoE or GERD. The determination of degranulation products at local level may therefore constitute a useful tool for the monitoring of the condition, although further studies would be warranted in order to assess this strategy.

Peripheral eosinophilia ( $>350$ eosinophils $/ \mathrm{mm}^{3}$ ), is frequently observed in EoE patients (1) and its role as a marker of activity has been assessed in more depth than the other two parameters studies herein. Baxi et al. (10) found a positive correlation between the number of eo/ hpf in biopsies and the presence of peripheral eosinophilia ( $35 \%$ in $<5$ eo/hpf vs. $67 \%$ in $>15$ eo/hpf; $\mathrm{p}=0.03$ ). 
Straumann et al. (9) also demonstrated how PBE levels drop after effective treatment with budesonide, and also correlate to the number of eosinophils found in the esophageal biopsy. Similarly, Konikoff et al. (21) also found a significant correlation between $\mathrm{PBE}$ and the number of eo/ hpf $(r=0.56, p<.0001)$ in 47 pediatric patients with EoE. All these findings partially match the results of our study where a significant drop in PBE is observed in responding $v s$. non-responding patients, albeit we did not find any significant correlation between count in blood and in biopsies. We can assume that this finding may be due, as in the case of the other two markers analyzed, to the influence of concomitant atopic conditions over this parameter.

To date, no studies have drawn a correlation between endoscopic findings and serological markers for activity in this condition. In this regard, we have found another aspect which supports the utility of PBE as a marker of EoE activity, as we observed how those patients presenting improved endoscopic results with the disappearance of signs of esophageal wall remodeling, express significantly less PBE. This correlation was not observed for the other two parameters studied. As regards the relationship between the clinical condition of patients measured using the ELSA index and markers for activity, we found no positive correlation. This is to be expected, as it is common for this condition to present dissociation between improvements in symptoms and histological response (13). However, we recognize the limitation that the ELSA index is as yet non-validated as an assessment method.

Not analyzing the period in which certain tests were performed, bearing in mind pollination seasons as per patient sensitivity (73.3\% sensitive to pneumoallergens) is a significant limitation in this study, as the parameters analyzed may vary based on this factor (22). Another limitation in the study design is the non-determination of these parameters during the reactivation phase of the condition in responding patients, which may provide a reliable indication of the role of PBE as a market of activity.

In conclusion, and in the light of the results obtained, serum levels of total IgE and ECP are not of use in monitoring EoE activity. However, PBE may play a role as a marker in this regard, although the high proportion of concomitant atopic conditions may affect this parameter, and results should be interpreted with caution. It is important to reproduce these results bearing in mind pollination seasons in order to check whether PBE does actually act as a marker of EoE activity and not merely as a marker of immunoallergic conditions. Given that our results were obtained with a small number of patients, further studies with larger patient groups should be completed.

\section{REFERENCES}

1. Liacouras CA, Furuta GT, Hirano I, Atkins D, Attwood SE, Bonis PA, et al. Eosinophilic esophagitis: Updated consensus recommendations for children and adults. J Allergy Clin Immunol 2011;128:3-20.
2. Prasad GA, Alexander JA, Schleck CD, Zinsmeister AR, Smyrk TC, Elias RM, et al. Epidemiology of eosinophilic esophagitis over three decades in Olmsted County, Minnesota. Clin Gastroenterol Hepatol 2009;7:1055-61.

3. Lucendo AJ, Sanchez-Cazalilla M. Adult versus pediatric eosinophilic esophagitis: important differences and similarities for the clinician to understand. Expert Rev Clin Immunol 2012; 8:733-45.

4. Lucendo AJ, Arias A, Gonzalez-Cervera J, Yague-Compadre JL, Guagnozzi D, Angueira T, et al. Empiric 6-food elimination diet induced and maintained prolonged remission in patients with adult eosinophilic esophagitis: A prospective study on the food cause of the disease. J Allergy Clin Immunol 2013;131:797-804.

5. Gupta SK. Noninvasive markers of eosinophilic esophagitis. Gastrointest Endosc Clin N Am 2008;18:157-67.

6. Cheng KJ, Xu YY, Liu HY, Wang SQ. Serum eosinophil cationic protein level in Chinese subjects with nonallergic and local allergic rhinitis and its relation to the severity of disease. Am J Rhinol Allergy 2013;27:8-12.

7. Rodríguez Sánchez J, Gómez Torrijos E, De La Santa Belda E, Martín Escobedo R, Martín Dávila F, López Viedma B, et al. VAS-EoE score: A new symptoms assessment tool for eosinophilic esophagitis. Gastroenterology 2013;144(5 Supl. 1):S-492 \#Su1855.

8. Dellon ES, Kim H, McConville S, Rybnicek D, Woosley JT, Shaheen NJ. Eosinophilic esophagitis is a progressive fibrostenotic disease: insights from a phenotypic analysis. Gastroenterology 2013;144(5 Supl. 1):S-486 \#Su1833.

9. Straumann A, Conus S, Degen L, Felder S, Kummer M, Engel H, et al. Budesonide is effective in adolescent and adult patients with active eosinophilic esophagitis. Gastroenterology 2010;139:1526-37.

10. Baxi S, Gupta SK, Swigonski N, Fitzgerald JF. Clinical presentation of patients with eosinophilic inflammation of the esophagus. Gastrointest Endosc 2006;64:473-8.

11. Vicario M, Blanchard C, Stringer KF, Collins MH, Mingler MK, Ahrens A, et al. Local B cells and IgE production in the oesophageal mucosa in eosinophilic oesophagitis. Gut 2010;59:12-20.

12. Mishra A, Schlotman J, Wang M, Rothenberg ME. Critical role for adaptive $\mathrm{T}$ cell immunity in experimental eosinophilic esophagitis in mice. J Leukoc Biol 2007;81:916-24.

13. Gonsalves N, Yang GY, Doerfler B, Ritz S, Ditto AM, Hirano I Elimination diet effectively treats eosinophilic esophagitis in adults; food reintroduction identifies causative factors. Gastroenterology 2012;142:1451-9.

14. Hastie AT, Moore WC, Li H, Rector BM, Ortega VE, Pascual RM, et al. Biomarker surrogates do not accurately predict sputum eosinophil and neutrophil percentages in asthmatic subjects. J Allergy Clin Immunol 2013; 132:72-80.

15. Petsky HL, Cates CJ, Lasserson TJ, Li AM, Turner C, Kynaston JA, et al. A systematic review and meta-analysis: tailoring asthma treatment on eosinophilic markers (exhaled nitric oxide or sputum eosinophils). Thorax 2012;67:199-208.

16. Blaiss MS. Epidemiology and pathophysiology of immunoglobulin E-mediated asthma. Allergy Asthma Proc 2005;26:423-7.

17. Rao G, Mitchell L, Ohnuki L, Fitzgerald J, Gleich G, Corkins M, et al Can eosinophil-derived neurotoxin (EDN) act as a surrogate marker of disease activity in children with allergic eosinophilic esophagitis (AEE)? Gastrointest Endosc 2004;59:103 \#465.

18. Dellon ES, Chen X, Miller CR, Woosley JT, Shaheen NJ. Diagnostic utility of major basic protein, eotaxin-3, and leukotriene enzyme staining in eosinophilic esophagitis. Am J Gastroenterol 2012;107:1503-11.

19. Chehade M, Yershov O, Sampson HA. Serum eosinophil cationic protein and eosinophil derived neurotoxin are potential non-invasive biomarkers for eosinophilic esophagitis. Gastroenterology 2007;132(4 Supl. 2):A-6.

20. Furuta GT, Kagalwalla AF, Lee JJ, Alumkal P, Maybruck BT, Fillon S, et al. The oesophageal string test: A novel, minimally invasive method measures mucosal inflammation in eosinophilic oesophagitis. Gut 2013 doi:10.1136/gutjnl-2012-303171.

21. Konikoff MR, Blanchard C, Kirby C, Buckmeier BK, Cohen MB, Heubi JE, et al. Potential of blood eosinophils, eosinophil-derived neurotoxin, and eotaxin-3 as biomarkers of eosinophilic esophagitis. Clin Gastroenterol Hepatol 2006;4:1328-36.

22. Yalcin AD, Basaran S, Bisgin A, Polat HH, Gorczynski RM. Pollen aero allergens and the climate in Mediterranean region and allergen sensitivity in allergic rhinoconjunctivitis and allergic asthma patients. Med Sci Monit 2013; 19:102-10. 